[6] M. González and J. M. Gutiérrez, Unconditionally converging polynomials on Banach spaces (to appear).

[7] A. Grothendieck, Sur les applications linéaires faiblement compactes d'espaces du type $C(K)$, Canad. Math. J. 5 (1953), 129-173.

[8] A. Pelczyński, A property of multilinear operations, Studia Math 16 (1957), 173-182.

[9] A. Pelczyński, Banach spaces on which every unconditionally converging operator is weakly compact, Bull. Acad. Polon. Sci. 10 (1962), 641-648.

[10] A. Pełczyński, On weakly compact polynomial operators on B-spaces with Dunford-Pettis property, Bull. Acad. Polon. Sci. 11 (1963), 371378.

[11] R. A. Ryan, Dunford-Pettis properties, Bull. Acad. Polon. Sci. 27 (1979), 373-379.

[12] R. A. Ryan, Applications of topological tensor products to infinite dimensional holomorphy, (Ph. D. Thesis, Trinity College, Dublin, 1980), 1980.

[13] R. A. Ryan, Weakly compact holomorphic mappings on Banach spaces, Pacific J. Math. 131 (1988), 179-190.

[14] B. S. Tsirelson, Not every Banach space contains an embedding of $\ell_{p}$ or $c_{0}$, Functional Anal. Appl. 8 (1974), 138-141.

\section{Manuel González}

Departamento de Matemáticas

Facultad de Ciencias

Universidad de Cantabria

Avda. de Los Castros s.n.

E-39071 Santander (Spain)

Joaquín M. Gutiérrez

Departamento de Matemática Aplicada

ETS de Ingenieros Industriales

Universidad Politécnica de Madrid

José Gutiérrez Abascal 2

E-28006 Madrid (Spain)

\section{Research Announcement}

\section{TAYLOR-MONOMHAL EXPANSIONS \\ OF HOLOMORPHIC FUNCTIONS ON FRECHET SPACES}

Seán Dineen

Let $\lambda:=\lambda(A)$ denote a Fréchet nuclear spaces with Köthe matrix $A$ and let $\left\{E_{n}\right\}_{n}$ denote a sequence of Banach spaces. Let $E:=$ $\lambda\left(\left\{E_{n}\right\}_{n}\right):=\left\{\left(x_{n}\right)_{n}: x_{n} \in E_{n}\right.$ and $\left.\left(\left\|x_{n}\right\|\right)_{n} \in \lambda(A)\right\}$ and endow $E$ with the topology generated by the semi-norms

$$
\left\|\left(x_{n}\right)_{n}\right\|_{k}:=\sum_{n=1}^{\infty} a_{n, k}\left\|x_{n}\right\|, \quad k=1,2, \ldots
$$

$E$ is a Fréchet space and $\left\{E_{n}\right\}_{n}$ is an unconditional Schauder decomposition of $E$. Examples of spaces which can be represented in this fashion, include all Banach spaces and all Fréchet nuclear (and some Fréchet-Schwartz) spaces with basis. Let $H(E)$ denote the space of all $\mathrm{C}$-valued holomorphic functions on $E$ and for $m \in N^{(N)}, m=\left(m_{1}, \ldots, m_{n}, 0 \ldots\right)$ let

$$
P_{m}(x)=\frac{1}{(2 \pi i)^{n}} \int_{\left|\lambda_{i}\right|=1} \frac{f\left(\sum_{i=1}^{n} \lambda_{i} x_{i}\right)}{\lambda_{1}^{m_{1}+1} \ldots \lambda_{n}^{m_{n}+1}} d \lambda_{1} \ldots d \lambda_{n}
$$

We have

$$
f=\sum_{m \in N^{(N)}} P_{m}
$$

in the $\tau_{0}, \tau_{w}, \tau_{\delta}$ topologies on $H(E)$

The expansion $(*)$ reduces to the Taylor series expansion in the case of a Banach space (i.e. if $E_{1}=E, E_{n}=0, n>1$ ) and to the monomial expansion for Fréchet nuclear spaces with a basis (when $\operatorname{dim}\left(E_{n}\right)=1$ all $n$ ). 
If $\left(E_{n}\right)_{n}$ is an unconditional Schauder decomposition for the Fréchet space $E$ then the topology of $E$ is generated by semi-norms satisfying

$$
\left\|\sum_{n=1}^{\infty} x_{n}\right\|=\sup _{\mid \lambda_{n} \leq 1}\left\|\sum_{n=1}^{\infty} \lambda_{n} x_{n}\right\|
$$

If $\left(\beta_{n}\right)_{n=1}^{\infty}$ is a sequence of real numbers, $\beta_{n} \geq 1$ all $n$, let

$$
\left\|\sum_{n=1}^{\infty} x_{n}\right\|_{\beta, j}=\left\|\sum_{n=1}^{j} x_{n}+\sum_{n=j+1}^{\infty} \beta_{n} x_{n}\right\|
$$

If $m \in N^{(N)}$ we let $\mathcal{P}_{e}\left({ }^{m} E\right)$ denote the set of all $|m|$-homogeneous polynomials on $E$ which are homogeneous in the even variables i.e. if $m=\left(m_{1}, m_{2}, \ldots, m_{n}, \ldots\right)$ then $P \in \mathcal{P}_{e}\left({ }^{m} E\right)$ if and only if

(i) $P(\lambda x)=\lambda^{|m|} P(x)$ for all $x \in E, \lambda \in \mathbb{C}$. (ii) $P\left(\lambda x_{2 i}+\sum_{n=1}^{\infty} x_{n}\right)=\lambda^{m_{2 i}} P\left(\sum_{n=1}^{\infty} x_{n}\right)$ for all $i$, all $x \in E$
and all $\lambda \in \mathbb{C}$.

Our main technical tool is the following proposition.

Proposition. Let $\left\{E_{n}\right\}_{n}$ denote an unconditional Schauder decomposition for the Fréchet space $E$, let $F$ denote a Banach space and let $T$ denote an $F$-valued linear function on $H(E)$ which is bounded on the locally bounded subsets of $H(E)$. Let $\beta_{n} \geq 1$ all $n, \beta_{2 n-1}=2$ all $n$ and suppose $\sum_{n=1}^{\infty} x_{n} \in E$ implies $\sum_{n=1}^{\infty} \beta_{n}^{p} x_{n} \in E$ for all $p>0$. Let $\|\cdot\|$ denote a continuous semi-norm satisfying (**) and suppose there exists $C>0$ such that

$$
\|T(P)\| \leq C\|P\| \quad \text { for all } P \in \mathcal{P}_{e}\left({ }^{m} E\right) \text { and all } m \in N^{(N)}
$$

where $\|P\|=\sup \{|P(x)| ;\|x\| \leq 1\}$. Then, for any $\delta>1$, there exists $C_{1}>0$ and a positive integer $j$ such that

$$
\|T(P)\| \leq C_{1} \delta^{|m|}\|P\|_{\beta, j}
$$

for all $P \in \mathcal{P}_{\mathrm{e}}\left({ }^{m} E\right)$ and all $m \in N^{(N)}$ where

$$
\|P\|_{\beta, j}=\sup \left\{|P(x)| ;\|x\|_{\beta, j} \leq 1\right\} .
$$

Theorem. Let $\lambda(A)$ denote a Fréchet-nuclear space with $D N$ and let $\left\{E_{n}\right\}_{n}$ denote a sequence of Banach spaces each of which admits an unconditional finite dimensional Schauder decomposition. Then $\tau_{w}=\tau_{\delta}$ on $\mathcal{H}\left(\lambda\left(\left\{E_{n}\right\}_{n}\right)\right)$.

A Fréchet nuclear space has $D N$ if and only if it is isomorphic to a subspace of $s$. The above theorem includes the known cases where $E$ is a Banach space with an unconditional basis and the case where $E$ is a Fréchet-nuclear space with basis and $D N$. It also includes Fréchet-Schwartz spaces which are not nuclear. By considering complemented subspaces, we find that $\tau_{w}=\tau_{\delta}$ on $\mathcal{H}(E)$, where $E$ is any reflexive subspace, with the approximation property, of a Banach space with an unconditional finite dimensional Schauder decomposition. This includes spaces which do not have a finite dimensional Schauder decomposition.

Seán Dineen,

Department of Mathematics,

University College Dublin,

Belfield,

Dublin 4. 